\title{
Tangence
}

\section{Québec : paradigme littéraire et éclosion culturelle à la fin du XVIIIe siècle}

\section{Bernard Andrès}

Numéro 51, mai 1996

Paradigmes critiques

URI : https://id.erudit.org/iderudit/025905ar

DOI : https://doi.org/10.7202/025905ar

Aller au sommaire du numéro

Éditeur(s)

Tangence

ISSN

0226-9554 (imprimé)

1710-0305 (numérique)

Découvrir la revue

Citer cet article

Andrès, B. (1996). Québec : paradigme littéraire et éclosion culturelle à la fin du XVIIIe siècle. Tangence, (51), 67-80. https://doi.org/10.7202/025905ar d'utilisation que vous pouvez consulter en ligne.

https://apropos.erudit.org/fr/usagers/politique-dutilisation/ 


\section{Québec : paradigme littéraire et éclosion culturelle à la fin du XVIII ${ }^{e}$ siècle}

\section{Bernard Andrès}

Comment parler au Québec de paradigme littéraire, d'éveil culturel ou de modernité dès la fin du xvIII siècle? Au mieux, voilà une anachronie douteuse, au pire une boutade sans conséquence. Linguistes et didacticiens nous opposeront le taux élevé d'analphabétisme et les conditions alarmantes de l'éducation à l'époque. Les historiens déploreront l'état de prostration dans lequel la Conquête et le Régime militaire anglais jetèrent alors les Canadiens. Les philosophes argueront de l'isolement de la "Province of Quebec " et sociologues et littéraires, de l'insuffisance des appareils éditoriaux ${ }^{1}$. Tous convaincus de l'incongruité de l'hypothèse, ils se tourneront délibérément vers la période contemporaine pour parler de véritable éclosion culturelle et interculturelle. Celle-ci ne daterait au plus que des trois ou quatre dernières décennies (après le Refus global, diront-ils). Quant à la "modernité", ajouteront-ils, le mot n'apparaît qu'en 1823 chez Balzac ${ }^{2}$. Alors, à quoi bon chercher au-delà, même si "le moderne" se retrouve chez Voltaire dès 1756 et "le moderniste" chez Rousseau dès 1769. Bataille de mots? Peut-être pas. Car la chose, elle, se précise bien à mes yeux dès les dernières décennies des Lumières, en Europe comme en Amérique du Nord. Et le Bas-Canada d'alors n'y échappe pas. C'est ce que j'entends montrer ici en examinant comment un paradigme littéraire encore

1 Sur cette époque et les problématiques évoquées dans cet article, voir la bibliographie d'Isabelle Beaulé dans Voix et images, $\mathbf{n}^{\circ} 59$, hiver 1995 , dossier "Archéologie du littéraire au Québec", p. 388-397. Sur la façon dont l'historiographie traditionnelle a perçu la Conquête, voir notamment François Xavier Garneau, Histoire du Canada depuis sa découverte jusqu'à nos jours, Québec, Aubin, 1845; Lionel Groulx, Lendemains de conquête. Cours d'bistoire du Canada à l'Université de Montréal, 1919-1920, Montréal, Bibliothèque de l'Action française, 1920; et Michel Brunet, La présence anglaise et les Canadiens. Études sur l'bistoire et la pensée des deux Canados, Montréal, Beauchemin, 1958.

2 Le Robert. dictionnaire bistorique de la langue française, 1992, p. 1258. Même référence pour les données lexicographiques suivantes. 
68

embryonnaire, certes $^{3}$, s'insère alors dans la modernité de son époque: les Lumières.

$$
\underset{*}{*}
$$

Qu'on soit souverainiste ou fédéraliste, qu'on veuille intégrer les minorités culturelles dans le creuset d'une identité québécoise ou qu'on voie dans la seule "mosaïque" canadienne une garantie pour ces minorités, la question de l'interculturel apparait d'abord comme une réalité contemporaine, j'en conviens. Le choc des cultures ou leur hybridation harmonieuse, les inquiétudes ou les espoirs suscités, les phénomènes d'acculturation ou de transculturation sont présentés par les sociologues, les historiens et les littéraires comme des faits plutôt récents, sinon résolument actuels. Qu'on les situe au tournant des années quatre-vingt, ou qu'on les fasse remonter à la Révolution tranquille et à "l'ouverture du Québec sur le monde", qu'on remonte à la deuxième Guerre mondiale ou à la première qui, chacune en leur temps, connurent d'importants flux migratoires, déplaçant des Québécois vers l'extérieur et de nouveau venus vers le Québec, qu'on tienne le présent immédiat ou le passé proche comme les moments clés d'une éclosion culturelle, le vingtième siècle reste pour beaucoup l'ère privilégiée du changement. Le $\mathrm{xx}^{\mathrm{e}}$ siècle serait l'espace de référence pour toute étude du renouveau socio-culturel au Québec, du modernisme au post-modernisme. Avant ce siècle, point de salut. Le $\mathrm{XIX}^{\mathrm{e}}$ siècle est alors perçu comme un éteignoir et les velléités d'indépendance de l'Institut canadien comme un épiphénomène négligeable en regard du courant dominant ultramontain.

Si ce point de vue apparaît discutable dans le détail (qu'on songe à la pensée libérale des Patriotes, aux Guibord, Dessaules, Buies et autres "rouges"), il reste que le siècle de $M^{\text {grs }}$ Lartigue et Bourget, de Tardivel et du juge Routhier ne figure pas dans les annales comme un exemple d'ouverture à l'Autre. Pour le nationalisme à la sauce ultramontaine (comme pour certaines variantes contemporaines), la culture de l'Autre est vécue comme un danger

3 Cf. mon article "Le texte embryonnaire ou l'émergence du littéraire au Québec, 1764-1815", La rechercbe littéraire. Objets et métbodes, Claude Duchet et Stéphane Vachon (dir.), Montréal, XYZ et Paris, Presses universitaires de Vincennes, 1993, p. 29-39. 
mortel: "Quocumque die comederis ex eo, morte morieris"; au jour où vous mangerez de ce fruit, vous mourrez. C'est ce que tonnait l'abbé Colin, prédicateur à Québec dans les années $1860^{4}$. Ce qu'il disait des mauvais livres valait mutatis mutandis pour toute manifestation culturelle marginale, pour toute importation douteuse d'hommes ou d'idées. Que penser aujourd'hui de cette entreprise forcenée (mais un peu vaine) destinée au XIX ${ }^{\mathrm{e}}$ siècle à contrer l'interculturel par l'institution d'un Index, de l'Euvre des bons Livres (1844), d'un Cabinet de lecture (1857), de bibliothèques paroissiales (1845) et autres cercles et revues sous influence cléricale? Yves Dostaler, Yvan Lamonde et d'autres ont montré l'efficacité toute relative de la chose ${ }^{5}$. Les écrits circulaient, tout comme certaines représentations de l'Autre par rapport auxquelles les Canadiens devaient eux-mêmes se (re)définir. Je pense à toutes ces préfaces de romans du XIX ${ }^{e}$ siècle où les auteurs du cru se comparent à leurs pairs européens ou s'en démarquent, les invoquent ou les révoquent tour à tour, se forgeant ainsi un caractère propre ${ }^{6}$.

Mais, demandons-nous, d'où part cette longue mutation qui transformera au cours du siècle les scripteurs anonymes en signataires d'une brochure, puis d'un volume, puis d'une oeuvre? Quelle est l'origine de ce passage obligé vers la fonction d'auteur ${ }^{7}$ ? Le $\mathrm{xIX}^{\mathrm{e}}$ siècle et le tournant du $\mathrm{xx}^{\mathrm{e}}$ siècle sont-ils seuls responsables du glissement entre écrivains canadiens et intellectuels québécois? Je crains qu'il faille remonter au-delà pour comprendre cette gestation culturelle et idéologique allant de Lacombe, Doutre, Marmette et de Gaspé à Garneau, Casgrain, Dessaules, Beaugrand, Buies et Laberge, puis au Nigog, à Cité libre, à l'Hexagone, Parti pris, Mainmise et La barre du jour (pour n'évoquer trop furtivement que quelques relais obligés). Je crains, oui, qu'il faille chercher au-delà de la Confédération, au-delà de I'Union,

4 Yves Dostaler, Les infortunes du roman dans le Québec du xIX siècle, Montréal, Hurtubise HMH, Les cahiers du Québec, 1977, p. 89 [176 p.].

5 Yves Dostaler, op. cit., Yvan Lamonde, Les bibliothèques personnelles au Québec. Inventaire analytique et préliminaire des sources, Montréal, Bibliothèque nationale du Québec, 1983, L'bistoire des idées au Québec 1760-1960 : bibliographie des études, Montréal, Bibliothèque nationale du Québec, 1989, et Louis-Antoine Dessaulles (1818-1895) Un seigneur libéral et anticlérical, Montréal, Fides, 1994.

6 Guildo Rousseau, Préfaces des romans québécois du xIX siècle, Sherbrooke, éditions Cosmos, 1970.

7 Cf. Robert, Lucie, L'institution du littératre au Québec, Québec, Ste-Foy, Les Presses de l'Université Laval; 1989, 272 p. 
pour saisir les prémisses d'une ouverture culturelle du Québec et d'une présence active de l'écrivain dans la cité. Au terme de ce préambule, passons donc à la fin du XvIII ${ }^{\mathrm{e}}$ siècle.

Sur la période d'intense activité culturelle, politique et intellectuelle de l'après-Conquête (de 1764 aux années 1790), nous commençons à en savoir davantage depuis le renouveau des études sur le $\mathrm{xvII}^{\mathrm{e}}$ siècle québécois. Depuis une quinzaine d'années, en effet, les travaux des historiens de l'imprimé ${ }^{8}$ ont relayé utilement les monographies de Camille Roy, Lionel Groulx, Marcel Trudel et Séraphin Marion (1953) ${ }^{9}$. Tour à tour, les publications sur Fleury Mesplet, Valentin Jautard, Pierre du Calvet et Pierre de Sales Laterrière, ainsi que l'entreprise La vie littéraire au Québec ${ }^{10}$ ont apporté de nouveaux éclairages sur les Lumières canadiennes ${ }^{11}$. Notre propre projet intitulé "Archéologie du littéraire au Québec" (ALAQ) et notre collection des "Cahiers de l'ALAQ" ont, je pense, contribué à mettre à jour les données sur cette époque

8 André Lamonde (dir.), L'imprimé au Québec. Aspects historiques (18e-20 siècles), Québec, Institut québécois de recherche sur la culture, 1983, 368 p.; Gilles Gallichan, Livre et politique au Bas-Canada, 1791-1849, Sillery, Editions du Septentrion, 1991, 519 p.; Narcisse-Eutrope Dionne, Inventaire chronologique des livres, brocbures, journaux et revues publiés en langue française dans la province de Québec, depuis l'établissement de limprimerie en Canada jusqu'à nos jours, 1764-1905, Québec, s. éd., 1905, 175 p.; Inventaire cbronologique des livres, brochures, journaux et revues publiés en langue anglaise dans la province de Québec, depuis l'établissement de limprimerie en Canada jusqu'à nos jours, 1764-1906, t. III, Québec, s. éd., 1907, viii-228 p., 4 vol.; Inventaire cbronologique des livres, brochures, journaux et revues publiés en diverses langues dans et bors la province de Québec, $1^{\mathrm{er}}$ supplément, 1904-1912, Québec, s. éd., 1912, 76 p.

9 Camille Roy, Nos origines littéraires, Québec, Imprimerie de l'Action sociale, 1909; Lionel Groulx, Lendemains de conquête. Cours d'bistoire du Canada à l'Université de Montréal, 1919-1920, Montréal, Bibliothèque de l'Action française, 1920; Marcel Trudel, LInfluence de Voltaire au Canada, t. I: 1760 à 1850, Montréal, Fides et Les Publications de l'Université Laval, 1945; Séraphin Marion, Les lettres canadiennes d'autrefois, Ottawa et Hull, Editions de l'Université d'Ottawa et les Éditions "l'Eclair", $2^{\mathrm{C}}$ édition, 1953, 2 vol.

$10 C f$. Maurice Lemire (dir.), La vie littéraire au Québec, $t$. 1: la voix française des nouveaux stuets britanniques, 1764-1805, Sainte-Foy, Presses de l'Université Laval, 1991, 498 p.; t. II: le projet national des Canadiens, 1806-1839, Sainte-Foy, Presses de l'Université Laval, 1992, 587 p.

11 Jean-Paul de Lagrave, Fleury Mesplet (1734-1794): diffiseur des lumières au Québec, Montréal, Patenaude éditeur, 1985; Jean-Paul de Lagrave et Jacques G. Ruelland, Premier joumaliste de langue française au Canada. Valentin Jautard, 1736-1787, préface de Jean-Louis Gagnon, Sainte-Foy, Éditions Le Griffon d'argile, 1989 et, des mêmes auteurs, Pierre du Calvet. Appel à la 
pionnière ${ }^{12}$. Dans le dossier que nous venons de publier à Voix et images, on trouvera un certain nombre d'études sur la Gazette littéraire de Montréal et sur des écrivains comme Joseph Quesnel, Pierre Roubaud, Luc Saint-Luc de La Corne ${ }^{13}$. Je ne reviendrai donc pas sur cette "génération de la Conquête" dont j’ai présenté les grandes lignes dans ce dossier. J'insisterai plutôt sur un aspect plus particulier du phénomène: la pluralité culturelle tout à fait nouvelle à laquelle fut alors confronté le Québec.

Pour bien comprendre l'impact de la Conquête sur la société canadienne, il faut se rappeler la période antérieure du régime français et la composition presque monolithique de la société canadienne d'alors. On sait que le type de colonisation pratiqué en Nouvelle-France ne favorisait nullement son expansion économique, démographique, politique ou culturelle (à l'inverse de ce qui se passait alors dans les colonies anglaises). Il en était de même aux plans religieux et ethnique, puisque les protestants en furent vite écartés et que le maigre flux migratoire, d'origine essentiellement hexagonale, ne permit pas à la société canadienne

justice de l'État, extraits, Sainte-Foy, Le Griffon d'argile, 1986. Sur Laterrière, cf. René Beaudoin, "Pierre de Sales Laterrière, médecin, mémorialiste et prototype de l'aventurier des lettres", dans Bernard Andrès, Principes du littéraire au Québec, 1766-1815, 1993, p. 43-56 et Bernard Andrès, "Statut de l'écrit intime dans une littérature en émergence: le cas des Mémoires de Pierre de Sales Laterrière (1743-1815),, Tangence, Université du Québec à Rimouski, no 45, octobre 1994́, p. 91-106.

12 Cf. Bernard Andrès et Pascal Riendeau (dir.), La Conquête des Lettres au Québec (1764-1815); Florilège, Montréal, UQAM, Département d'Études littéraires, Cahiers de l'ALAQ, ${ }^{\circ} 1$, mars 1993; Bernard Andrès, Principes du littéraire au Québec (1766-1815), Cahiers de l'ALAQ, $\mathrm{n}^{\circ} 2$, août 1993; Bernard Andrès et Pierre Lespérance (dir.), Fortune et infortunes d'un dandy canadien. Pierre-Jean de Sales Laterrière: Journal de voyage (1815), Cahiers de l'ALAQ, $\mathrm{n}^{\circ} 3$, hiver 1994, et, des mêmes éditeurs, En quête d'origines. PierreJean de Sales Laterrière: Nouveaux journaux de voyage $(1824,1826,1827$ \& 1829), Cahiers de l'ALAQ, no 4, été 1995.

13 Voir dans Voix et images, $\mathrm{n}^{\circ}$ 59, hiver 1995: Bernard Andrès, "La génération de la Conquête: un questionnement de l'archive „, Pierre Hébert et Jacques Cotnam, "La Gazette littéraire de Montréal (1778-1779): notre première cuvre de fiction?", p. 294-312; Caroline Masse, "Pierre Roubaud, polygraphe et faussaire au Siècle des Lumières", p. 314-328; Pierre Lespérance, "La fortune littéraire du Journal de voyage de Saint-Luc de La Corne", p. 329-341; Robert Derome, "Notes sur le pictural et le littéraire aux $\mathrm{XVIII}^{\mathrm{e}}$ et $\mathrm{XIX}{ }^{\mathrm{e}}$ siècles: le cas de Saint-Luc de Lacorne", p. 342-347; John Hare, "Aperçus de la correspondance de Joseph Quesnel ", p. 348-361 et Lucie Robert, "Monsieur Quesnel ou le Bourgeois anglomane", p. 362-387. 
de se construire en fonction d'un Autre bien identifiable. À un certain degré, seuls l'administrateur, le clerc ou l'officier métropolitains pouvaient à la fin du régime français figurer l'Autre du Canadien de souche. Quant à l'Amérindien dont on a beaucoup glosé à propos des coureurs de bois et des rapports d'altérité qui purent s'établir entre eux, ni les statistiques démographiques, ni les travaux généalogiques ne révèlent de croisements significatifs sur la longue durée. Le phénomène des coureurs de bois et des "truchements " prenant contact avec les sociétés amérindiennes, faisant le pas vers l'Autre par la langue, les coutumes, etc., est un épiphénomène limité. Il concerne des individus marginalisés par le pouvoir colonial. Il en est de même des contacts épisodiques des troupes françaises avec les tribus alliées dans les guerres contre l'Angleterre. C'est surtout aux plans symbolique, littéraire, théologique et philosophique que se créeront (souvent ultérieurement), des "figures de l'Indien "14: bons ou maudits sauvages des écrits philosophiques ou religieux, héros ou anti-héros des romans du XIX siècle, etc. J'ai montré ailleurs comment la représentation du sauvage dans le théâtre en Nouvelle-France supposait une abstraction de celui-ci; elle conduisait en fait à un effacement pur et simple du réel amérindien de la scène (scène dramatique et scène socioculturelle) ${ }^{15}$. Une forme de monolithisme caractérise donc la société canadienne au moment de la Guerre de sept ans: démographiquement et religieusement, on l'a vu, mais aussi linguistiquement (la francisation des pratiques s'effectue plus vite ici qu'en France), professionnellement (avec l'atténuation ou l'abandon des corporations de métiers) et même socialement (avec la porosité bien connue des systèmes de classes en Nouvelle-France).

D'où le mouvement de bascule qui se produit à la Conquête quand de vrais étrangers, d'Autres avec un grand A envahissent réellement la scène. Fernand Dumont a bien souligné l'importance de cette rupture au plan culturel, pour les Canadiens:

[...] la métropole [anglaise] représente d'autres coutumes, un autre langage, une autre religion. Les tentatives constantes d'as-

14 Cf. Gilles Thérien (dir.), Les figures de l'Indien, Montréal, Université du Québec à Montrêal, Les Cahiers du département d'études littéraires, $n^{\circ} 9$, 1988, 401 p.

15 Cf. Bernard Andrès, "Jouer le Sauvage: rôle, fonction et représentation de l'Indien dans les spectacles de Nouvelle-France ", dans Les figures de l'Inctien, op. cit., p. 163-180. 
similation et l'édification d'une société parallèle ne peuvent qu'accentuer chez les francophones le sentiment de la différence en même temps qu'une conscience plus vive de l'originalité de leurs propres institutions. ${ }^{16}$

J'ai déjà fait remarquer l'intérêt de cette analyse, car Dumont reste sensible à la "bigarrure des situations" et au "flottement des attitudes " observés à l'époque ${ }^{17}$. J'ajoute que ces "étrangers " qui considèrent eux-mêmes les Canadiens comme des "étrangers" sur leur propre sol, puisqu'ils les traitent de "nouveaux sujets", ces "Autres", donc, forcent les Canadiens à se sentir pour la première fois "étrangers à eux-mêmes" (pour paraphraser Julia Kristeva): l'invasion anglaise me semble alors vécue plus globalement comme une invasion généralisée. L'Autre ne présente plus alors un visage unique, mais, systématiquement, il représente l'image démultipliée du "Cosmopolitain", du "Citoyen du monde" cher à Fougeret de Monbron ${ }^{18}$. Par rapport à l'Autre qu'était jadis l'Amérindien, cet "autre Autre" de la Conquête (si j'ose dire), pourtant semblable par la couleur de la peau et l'origine européenne, reste intrinsèquement distinct: par la langue, la culture, la religion. C'est l'Anglais, l'Écossais, l'Irlandais, le loyaliste américain, mais aussi, sous l'habit rouge, les mercenaire suisses ou allemands, etc. Il faut aussi rappeler à cette époque le retour des huguenots français dont le mouvement s'était amorcé dès la fin du régime antérieur, mais qui s'accentue avec l'arrivée des Britanniques.

Et, contrairement aux Amérindiens de la Nouvelle-France, ces Autres avec un grand $\mathrm{A}$ ont le pouvoir avec un grand $\mathrm{P}$ : militairement, économiquement, administrativement et bientôt, constitutionnellement. On n'est plus dans l'ordre du fantasme, du symbolique, du littéraire. À partir de la Conquête, une société multiculturelle s'impose et il faut "faire avec". Se redéfinir avec ou contre (et, quelque part, grâce à) elle.

$\mathrm{Ce}$ que je maintiens, c'est qu'une telle redéfinition des rapports d'altérité, loin de nuire à la communauté canadienne, lui a fourni pour la première fois l'occasion de se questionner

16 Fernand Dumont, Genèse de la socièté québécoise, Montréal, Boréal, 1993, p. 117.

17 Voir mon article "La génération de la Conquête : un questionnement de l'archive", loc. cit., p. 277-278.

18 Fougeret de Monbron, Le Cosmopolite ou le Citoyen du monde. Suivi de la Capitale des Gaules ou la Nouvelle Babylone (1750), Bordeaux, Ducros, 1970. 
profondément et de prendre en charge son destin. Et, pour la première fois aussi, de le faire sur la place publique: dans "l'espace public" des gazettes, des pétitions, des lettres ouvertes et des sociétés de débats (clubs constitutionnels, salons, académies, loges maçonniques ${ }^{19}$, etc.). Car jusqu'alors veuve d'imprimerie, la société des "nouveaux sujets" britanniques se voit désormais dotée de presses qui, bien vite, diffuseront auprès des élites, mais aussi des populations campagnardes les idées du temps. Rappelons en effet que, sous le Rëgime français, la diffusion des Lumières n'avait atteint qu'une très petite caste d'administrateurs, d'officiers, etc. ${ }^{20}$ Ces idées n'étaient alors discutées que dans les salons. Avec la propagande et l'invasion américaines des années 1775-1776, pour la première fois ces idées atteignent des franges plus larges de population avec la diffusion dans les villes comme dans les campagnes, dans des réseaux de discussion tout à fait inédits ${ }^{21}$. Il s'agit de presses tenues d'abord par des anglophones, d'un journalisme adoptant le bilinguisme et dont le secret dessein d'assimiler les conquis ne résista pas longtemps à la réalité démographique, ni aux réactions des premiers intellectuels du temps.

Dès 1764, apparaissent en effet au Québec la première imprimerie et le premier hebdomadaire (bilingue), La Gazette de Québec/The Quebec Gazette. Le "prospectus" annonçant la parution évoque, dans un langage illustrant déjà les ravages du bilinguisme, les avantages linguistiques et transculturels d'une telle entreprise. C'est pour les éditeurs

[...] le Moyen le plus efficace a faire réussir une entière Connaissance de la Langue Angloise et Française parmi ces deux Nations, qui à présent se sont jointes heureusement dans cette Partie du Monde, ils seront rendus capables de converser ensemble, de se communiquer leurs Sentiments comme des Frères, et de conduire leurs différents Négoces avec Aise et Satisfaction. ${ }^{22}$

19 Roger Le Moine, "Francs-maçons francophones de la "Province of Quebec" ", in Cabiers des Dix, volume 48, 1993, p. 87-118.

20 Marcel Trudel, L'influence de Voltaire au Canada, op. cit., t. 1, p. 19-35.

21 Gustave Lanctôt (Le Canada et la Révolution américaine, 1774-1783, Montréal, Librairie Beauchemin, 1965) l'a bien montré, tout comme, à l'époque, les journaux de Badeaux et de Sanguinet. Voir aussi mon article "Le relais américain dans l'émergence des lettres au Québec (1764-1793)", in Bernard Andrès, Principes du littéraire au Québec, Cahiers de l'ALAQ, n ${ }^{\circ} 2$, UQAM, août 1993, p. 57-65.

22 Cité par Maurice Lemire (dir.), La vie littéraire au Québec, t. 1, op. cit., p. 228. 
Ce qui frappe l'observateur de cette période, c'est la faculté d'adaptation des pouvoirs et des contre-pouvoirs en place et les transferts culturels qui s'opèrent alors. L'adaptation d'abord. Les Britanniques sont bien vite convaincus de ne pouvoir mater les conquis et leur accordent la liberté du culte, de la langue et d'un certain espace juridique dans l'Acte de Québec (1774). Les Américains révoltés, eux, comprennent que Londres n'accorde ces privilèges aux Canadiens que pour mieux les dissuader de tenter l'aventure indépendantiste. Aussi, adaptent-ils leur propagande en fonction des publics visés: discours anti-papiste à l'intention des Anglais d'Angleterre, discours anti-britannique à l'intention des Canadiens ${ }^{23}$. Quant à ces derniers, ne s'adaptent-ils pas habilement à ces discours de séduction que leur adressent les anglophones de tous bords et ne tirent-t-ils pas parti de la situation en inquiétant aussi bien les uns et les autres, pour obtenir enfin une double reconnaissance (comme "peuple" et comme "nation" dans les déclarations que leur envoient les Américains)?

Pour ce qui est des transferts culturels à l'œuvre dans cette période, ils donnent un peu le vertige. Qu'on songe à cette ère fertile en bouleversements de toutes sortes: trauma de la Conquête, régime militaire, Acte de Québec, guerre d'indépendance américaine, invasion du Canada, siège de Québec, Constitution de 1791 et contrecoups de la Révolution française! Trente ans d'un climat éminemment volatile, où une ancienne culture britannique servie par des mercenaires germano-suisses en rencontre une autre, française, matinée de canadianité et qui se cherche elle-même au voisinage d'une autre culture naissante, l'américaine. Ces Américains sont eux-mêmes nourris des idéaux philosophiques français dont, les premiers, ils traduiront les principes jusqu'à leur ultime conséquence, la Révolution. Quand on s'avise, d'une part, que ces mêmes idéaux ont transité par l'Angleterre (lieu de prédilection des Philosophes en exil, modèle de régime parlementaire), et que, d'autre part, la France appuyant l'Amérique

23 Alors que la Lettre aux Canadiens du 26 octobre 1774 minimise les éventuels problèmes engendrés par "la diversité de religion "(catholique et protestante), une autre adresse du même Congrès américain datée du 21 octobre 1774, mais destinée, elle, au peuple anglais s'étonne "qu'un parlement britannique ait consenti à établir [au Québec] une religion qui a inondé de sang votre île et qui a répandu l'impiété, la bigoterie, la persécution, le meurtre et la rébellion dans toutes les parties du monde" ( $c f$. Latouche et Poliquin-Bourassa, Le manuel de la parole, t. 1, 1760-1891, Éditions du Boréal-Express, 1977, p. 33). 
76

y trouvera bientôt l'alliée mais, aussi, l'inspiratrice de sa propre Révolution (Franklin, Lafayette, Thomas Paine), quand on croise l'ensemble de ces variables idéologiques, on ne peut que constater la richesse culturelle éblouissante de cette époque pour un Québec à peine sorti de la léthargique Nouvelle-France.

Il va sans dire que cette volatilité des régimes et des événements, ainsi que l'hybridisation des systèmes de représentation et d'interprétation du réel, se reflète parfaitement dans les agents politiques et culturels alors impliqués sur le terrain. Je ne peux ici évoquer toutes ces grandes figures de l'époque et leurs parcours institutionnels, qui dans l'appareil britannique, qui dans les réseaux francophones ${ }^{24}$. Les textes qu'ils nous en ont laissé témoignent tous de ces transferts interculturels qui ne sont pas que des jeux d'influence (au sens traditionnel du terme). C'est dans la perspective foucaldienne des formations discursives et d'un certain ordre du discours, que s'apprécient pleinement ces pratiques culturelles ${ }^{25}$, un ordre discursif qui fait la part belle au désordre (apparent) de tel ou tel énoncé recueilli chez un politique, chez un religieux, chez un notable, un journaliste ou un pamphlétaire de l'époque.

Quelques exemples pour finir. Parmi toutes les personnalités de cette époque, se détache (positivement ou non selon les points de vue) la figure du génêral Frederick Haldimand ${ }^{26}$. Impliqué en Amérique du Nord depuis la Guerre de sept ans, il se retrouve gouverneur de la province du Québec entre 1778 et 1784, période tumultueuse de la guerre avec les colonies américaines. Suisse roman représentant le pouvoir britannique, il lance la première bibliothèque publique où figurent en bonne place les Philosophes et il tolère la première gazette littéraire... dont il incarcère pourtant les artisans durant près de quatre années, sans autre forme de procès ${ }^{27}$. C'est que la guerre fait rage avec les

24 Je prépare un essai sur le sujet.

25 Sur ces questions de méthode, voir dans mon article "La génération de la Conquête : un questionnement de l'archive", loc. cit., p. 278-285.

$26 C f$. Stuart R. J. Sutherland, Pierre Tousignant et Madeleine Dionne-Tousignant, "Haldimand, sir Frederick (baptisé François-Louis Fréderic)", in Dictionnaire biograpbique du Canada, Presses de l'Université Laval et University of Toronto Press, volume V, 1983, p. 977-995.

27 Rappelons que Haldimand, Suisse roman à la solde des Anglais, n'obtiendra jamais lui-même la citoyenneté britannique et qu'il aura lui-même affaire à l'intransigeance et à l'ingratitude de ses maîtres (il faut lire son journal et sa 
Bostonnais et qu'on soupçonne le journaliste Valentin Jautard, l'imprimeur Fleury Mesplet et quelques autres embastillés de n'être insensibles ni aux sirènes américaines, ni aux sémaphores des Lumières ( $s i$ j'ose dire). Chacun de ces prisonniers d'État a connu un parcours étonnant, de la province française aux capitales européennes et à l'Amérique, des Illinois au Massachusetts et à la Pennsylvanie. Désirée ou non, leur destination dernière, le Québec, devient vite leur port d'attache et leur terrain privilégié d'intervention. C'est de façon un peu fortuite que, par exemple, Valentin Jautard se rabat sur Montréal. Ce Bordelais d'origine y arrive dans la jeune trentaine, après un séjour aux Illinois. D'abord avocat, puis notaire des Fils de la Liberté, il sympathise avec les Bostonnais en 1775-1776 (accueillant en héros le général Montgomery à son arrivée à Montréal). Il s'associe ensuite avec Fleury Mesplet, lui aussi français d'origine et imprimeur du Congrès, qui fonde la première gazette francophone au Canada (ainsi que l'Académie voltairienne de Montréal") ${ }^{28}$. Tout en discutant de science, d'éducation, de philosophie et de littérature, Jautard invite les lecteurs à lui envoyer des poèmes qu'il publie, commente et corrige, donnant lui-même l'exemple (sous le pseudonyme du "Spectateur tranquille") 29 .

D'autres émigrants s'installent à la même époque sur les rives du Saint-Laurent pour y faire fortune, certes, mais aussi pour $y$ semer les germes d'un nouvel état d'esprit, celui des Lumières: dénonciation de l'injustice, tolérance et liberté de pensée. C'est là "l'esprit français" dont parlera $M^{\text {gr }}$ Camille Roy dans Nos origines littéraires. Commentant à sa façon cette époque d'éclosion culturelle, Camille Roy ne sera pas tendre pour les voltairiens du cru:

L'esprit français était malheureusement représenté par ces hommes à réputation louche, par ces demi-lettrés et par ces épaves de la morale que le flot de la mer avait déjà jeté sur nos rivages. ${ }^{30}$

correspondance pour apprécier correctement sa stratégie dans les années 1778 1782). Son rôle n'est pas si noir que cela dans la mesure où, en enfermant sans procès les intellectuels francophones jusqu'à la fin de la guerre avec les ÉtatsUnis, il ne les pend pas, et ce, pour ne pas en faire des martyrs et pour d'autres raisons, dont l'affiliation possiblement maçonnique de certains d'entre eux. Cf. Jean-Paul de Lagrave, Fleury Mesplet (1734-1794); diffuseur des lumières au Québec, op. cit., p. 127 sq.

29 Sur l'aventure éditoriale de ce journal, voir Pierre Hébert et Jacques Cotnam, loc. cit., supra, note 13 .

30 Camille Roy, Nos origines littéraires, 1909, op. cit., p. 68. 
J'ai montré ailleurs que, malgré tout, l'historien catholique avait bien saisi, pour son époque, le défi et les enjeux transculturels de la fin du XVII ${ }^{\mathrm{e}}$ siècle $^{31}$. Voici comment il caractérise en 1909 la dialectique des années 1770-1780 entre l'“esprit canadien" ou "national" et l'" esprit français":

C'est donc cet esprit [le français] qui apparaît, se manifeste et s'exprime dans plusieurs pages de la Gazette littéraire; comme l'on voit aussi protester dans ces mêmes pages l'esprit de ceux qui étaient nôtres, l'esprit national. Nul doute, poursuit-il, que l'esprit national n'ait plus d'une fois bénéficié de sa rencontre avec l'esprit français. Ces deux esprits ne pouvaient être, ils ne seront jamais, c'est du moins notre espérance, absolument opposés et contraires.

Je n'ai guère le loisir de montrer ici comment des Canadiens de souche furent eux-mêmes marqués par cette dialectique. Isabelle Beaulé vient de déposer un mémoire de maîtrise sur Henri-Antoine Mézière, jeune révolutionnaire qui aiguisa sa plume dans les colonnes de cette gazette, puis dans des correspondances privées et politiques et jusque dans un pamphlet qu'on lui attribue volontiers: La Bastille septentrionale (paru chez Fleury Mesplet en 1791) ${ }^{32}$. D'autres collaborateurs de l'ALAQ ont publié sur ces individus hauts en couleur: Pierre Lespérance sur Luc Saint-Luc de La Corne; Caroline Masse sur Pierre Roubaud; René Beaudoin sur Pierre de Sales Laterrière ${ }^{33}$. Julie Roy prépare une thèse sur les écrits de femmes à cette époque ${ }^{34}$. Quant à Osée Kamga, il prépare actuellement un mémoire sur l'intertexte des Lumières dans les écrits religieux de $\mathrm{M}^{\mathrm{gr}}$ Joseph-Octave Plessis. Joanne Gagnier en fait autant dans les textes de CharlesFrançois Bailly de Messein. Ce dernier, lui aussi Canadien d'ori-

31 Bernard Andrès, "La réception de l" étrange" au Québec. Pierre de Sales Laterrière (1743-1815)", dans Annette Hayward et Agnès Whitfield (dir.), Critique et littérature québêcoise, Montréal, Triptyque, 1992, p. 199-216.

32 Isabelle Beaulé, "Henri-Antoine Mézière: d'épistolier à pamphlétaire?n, mémoire de maîtrise (Bernard Andrès, directeur), UQAM, Département d'études littéraires, janvier 1996.

33 Voir supra, note 11. Yvan Brais a aussi étudié les journaux du notaire Badeaux et de l'instituteur Labadie et Bertrand Laverdure s'est intéressé pour sa part à la sensibilité préromantique de $\mathrm{M}^{\mathrm{gr}}$ Plessis dans ses récits de voyage (Dossiers de recherche, Archives de l'ALAQ, UQAM : 1994-1995).

34 Julie Roy, "Stratégies épistolaires et écritures féminines : les Canadiennes à la conquête des lettres (1759-1837)", thèse de doctorat (Bernard Andrès directeur), Département d'études littéraires, UQAM (en cours). 
gine, éduqué à Paris, puis missionnaire en Acadie, fut précepteur à Londres et ardent défenseur d'une université laïque en 1790 . Ce bouillant coadjuteur n'hésita pas à prendre le contre-pied de son évêque, $M^{\text {gr }}$ Hubert, dans une lettre ouverte d'une audace et d'une ironie toute voltairiennes. C'est à titre de "Canadien attaché à sa patrie par les liens les plus étroits ", qu'il appelle de ses voeux la création d'une institution de haut savoir où professeront des "hommes sans préjugés" et d'où l'on chassera les "fanatiques, monstres plus à craindre que tous ceux que produisent les déserts de l'Afrique". Il n'est pas indifférent que Bailly de Messein salue alors le "citoyen illustre", Simon Sanguinet, franc-maçon notoire qui dota à sa mort l'université projetée. Si l'Église et la famille Sanguinet empêchèrent par la suite ce don, Bailly de Messein n'en reconnaît pas moins la beauté du geste et "l'éclatant témoignage que le canadien ne soupirent (sic) qu'après une bonne éducation, et ne le cède point à nos voisins dans l'amour et le zèle du bien public". S'agit-il des voisins américains ou des Anglais du Canada (on sait que James McGill, autre philanthrope, devait doter en 1811 le collège qui deviendrait en 1821 l'Université McGill ${ }^{35}$ )? Quoi qu'il en soit, Bailly de Messein s'avère dans cette affaire comme à d'autres occasions le modèle même du Canadien éclairé, la preuve flagrante de l'éveil culturel de la province dix ans après les éclats de la Gazette de Montréal.

Avait collaboré à cette gazette éphémère un Français - le plus combatif de tous, assurément -, Pierre du Calvet, sur qui j'achèverai cette présentation. Lui aussi perçu comme un conspirateur, il fut jeté en prison d'où il ressortit, plus enragé que jamais, pour publier à Londres son pamphlet Appel à la justice de l'État (1784). Ce négociant qui entama sa carrière nord-américaine en Acadie sous le régime français, la poursuivit sans problème sous les Britanniques qui le nommèrent juge de paix. Mais au lieu de profiter de sa fortune et des avantages attachés à sa fonction, il se mit en tête de dénoncer les exactions de ses confrères magistrats, puis de réclamer à hauts cris un gouvernement responsable (ses projets de réforme conduisirent à la constitution de $1791^{36}$ ). Là encore, nous avons affaire, si j'ose dire, à un curieux OTNI (objet

35 Cf. John Irving Cooper, "McGill, James", Dictionnaire biographique du Canada, Presses de l'Université Laval et University of Toronto Press, volume V, 1983, p. 579-583.

36 Comme le rappelle justement Fernand Dumont en saluant l'apport de du Calvet dans la naissance d'une opinion publique: "Le livre [L'Appel ] connaîtra 
transculturel non identifié): natif de Caussade, près de Montauban, du Calvet vient d'un milieu protestant converti. De Lagrave et Ruelland ${ }^{37}$ rappellent que son ancêtre huguenot, François Calvet, périt sur l'échafaud en 1563. Élevé dans la religion catholique, Pierre du Calvet, nous dit-on, "conserva son attachement à la foi protestante . C'est ce qui apparaît clairement à la clôture de son pamphlet, qui témoigne bien d'une ouverture transculturelle qu'il brûle de transmettre aux Canadiens:

Ah! je suis protestant; mais au moins dans ma publication, j'ai suivi des principes d'équité bien différents, et qui sont de toutes religions: lisez les termes honorables sur lesquels je cite la vôtre; avec quel respect je fais mention de votre clergé et de vos communautés; avec quelle droiture je rends justice à leurs vertus; et avec quelle chaleur enfin je soutiens et défends leurs droits nationaux et même religieux $[\ldots]^{38}$

Et, dans ce texte tout empreint des idéaux philosophiques et même révolutionnaires - les Américains viennent d'obtenir leur indépendance - il conclut par un vibrant appel aux Canadiens. Je terminerai moi-même sur ce propos dont on jugera différemment de l'actualité selon qu'on y lira le destin du Québec ou celui du Canada :

[...] c'est à vous maintenant à vous défendre vous-mêmes. Si, imitant le passé, vous êtes les spectateurs oisifs et insensibles des événements, eh bien! votre province va être pour longtemps confirmée dans son esclavage jusqu'à ce que le désespoir au moins lui suscite des vengeurs [...] c'est à vous à voir s'il vous convient de vivre dans un vrai coupe-gorge où personne ne peut être un seul moment assuré de sa fortune, de sa liberté, de son honneur et de sa vie. J'ai des idées trop nobles de vos sentiments pour imaginer qu'une si terrible destinée puisse jamais être de votre goût. ${ }^{39}$

une large audience. Du Calvet est parvenu à composer une doctrine qui ralliera un assentiment quasi général de la part de ceux qui désirent des institutions représentatives; au surplus, il trace l'essentiel du programme qui sera pour longtemps celui des leaders canadiens après la venue de la Constitution de 1791 ", dans Genèse de la société quêbécoise, l'Hexagone, 1993, p. 142.

$37 C f$. Jean-Paul de Lagrave et Jacques G. Ruelland (éd.), Pierre du Calvet. Appel à la justice de l'Etat, extraits, op. cit., p. 1.

38 Ibid., p. 48-49.

39 Ibid., p. 49. 